\title{
Experimental modification of the lag between perceiving and performing
}

\author{
HELEN L. BEE AND ROBERTA S. WALKER, \\ DEVELOPMENTAL PSYCHOLOGY LABORATORY, \\ UNIVERSITY OF WASHINGTON, Seattle, Wash. 98105
}

Two types of discrimination training were used in an attempt to improve the drawing of geometric figures by preschool children. One type of training produced a significant improvement in drawings, while the other produced a significant decrement. The results suggest that some, but not all, of the "lag" between perceiving and performing may be due to insufficient discrimination.

It has seemed clear from a number of sources of evidence that young children make perceptual discriminations which they are unable to match in their own copying behavior. This has been observed in speech (Berko \& Brown, 1960; Metcalfe, 1962), where a child may misarticulate words he discriminates in others' speech, and in drawing (Piaget \& Inhelder, 1956; Maccoby \& Bee, 1965; Lovell, 1960), where a child is able to make discriminations between simple geometric figures such as circles and triangles before he is able to draw the figures accurately.

While there are a number of alternative explanations of this phenomenon, the present study focuses on one hypothesis, namely the "number of attributes" hypothesis suggested in an earlier analysis of the problem (Maccoby \& Bee, 1965). Stated most simply this hypothesis suggests that the number of attributes which must be noticed for discrimination of two distinct figures is smaller than the number required to make a good copy. To discriminate a circle from a triangle, the child must only notice that one is an angular figure and the other is not. But to draw both he must have attended to the length of lines, the nature of angles, the number of lines and angles, and the relationships between the different lines and angles. Effectively such an hypothesis suggests that there may be no "lag" at all; the child may quite simply be drawing what he has discriminated.

If this hypothesis is correct, it follows that discrimination training which focuses on the more detailed features of a figure should lead to better drawings. The present study is an attempt to test that deduction.

Subjects. Thirty four- and five-year-old children, all enrolled in a Head Start preschool program in Seattle, Washington, served as Ss for the complete experiment. Testing by one of three female Es was conducted at the Head Start center.

Procedure. The children were tested individually, each being given an initial discrimination test, a test of the ability to trace the stimuli, a drawing test, discrimination training, and finally a posttest of drawing ability. Two experimental groups ( $\mathrm{N}=10$ for each group) received slightly different discrimination training on one of the test figures while a control group $(\mathrm{N}=$ 10) received discrimination training on a different dimension.

In the initial discrimination test $S$ was presented with two large cardboard "mailboxes," labelled "Mr. Circle" and "Mr. Triangle." A white circle or triangle was affixed to the top of the box, below which was a slot through which stimulus cards could be "mailed." $S$ was given a set of eight randomly ordered cards including four triangles and four circles, and asked to "mail" them to Mr. Circle and Mr. Triangle.

Following the discrimination test, $\mathbf{S}$ was asked to trace two triangles and two circles, and was then asked to draw, freehand, four circles and four triangles. For each drawing, $S$ was shown a model, and asked to draw a picture that looked just like it. Only Ss who could discriminate and trace but who could not draw adequately were retained for the remaining portion of the experiment. Eight Ss whose drawings were too proficient were replaced.

Discrimination training for both experimental groups focused only on the triangle. Ss were presented with a partially programmed series of pairs of line drawings. Each pair contained one of six different triangles, and one of nine nontriangles. The nontriangles were designed to direct the child's attention to four requirements of a triangle, namely, that it have three sides, be enclosed, have pointed rather than rounded corners, and straight rather than curved sides. Accordingly, each nontriangle contained one kind of error, e.g., rounded sides, rounded angles, missing angles, a missing side, or an excess of lines. The pairs were presented in five blocks of trials, with $S$ required to achieve a criterion of six consecutive correct trials in each block. Thus each $\mathrm{S}$ received a minimum of 30 training trials.

For one training group, the "pointing" group, $S$ was required only to point to the correct item of the pair. Ss in the "tracing" group were also required to trace around the correct figure with their finger.

Control training followed the same procedure except that color rather than shape was the criterial attribute. Colored cutouts of rabbits were used as stimuli, with $\mathrm{S}$ required to pick the red stimulus on each trial.

Following training, $S$ was again asked to draw four triangles and four circles.

Results. To assess change in drawings, all pre- and posttest triangle drawings were independently scored by both authors. For each drawing, the number of errors in each of the four features emphasized in training were simply counted. That is, an error was scored for each extra line, each line fewer than three, each curved line, each curved angle, and each gap in the drawing. The interrater reliability of these ratings was .89. All disagreements were discussed, and a joint rating agreed upon.

For each $\mathrm{S}$ a mean error score for the four pre- and the four posttest triangle drawings was computed, and a difference score obtained. These difference scores were then compared by means of $t$ tests. The results of this analysis are presented in Table 1.

The results indicated a significant improvement in the pointing group, with a significant deterioration in the tracing group.

Discussion. Our results indicate a significant improvement in one group, and a significant deterioration in the other. The pointing group showed improvement in exactly those areas which were emphasized in training, which lends some support to the original hypothesis. For this group, discrimination training improved drawing. However, the deterioration in the tracing group runs counter to the original hypothesis.

The behavior of the tracing group can be explained most simply on the basis of fatigue. This experimental condition took over half again as long as the other procedures since Ss had to trace the triangle on each trial. The total testing time for this group was approximately $25 \mathrm{~min}$, while the remaining groups took only about $15 \mathrm{~min}$. All Es reported that by the time posttest drawings were required of the tracing Ss, they appeared tired and distracted, and their drawings hurried. Assuming that a fatigue interpretation is correct, then the results from the pointing group provide a clearer test of the original hypothesis.

There is, however, another discomforting feature of our findings, namely the fact that the experimental Ss made very few errors on the discrimination training trials. Overall, there were only 43 errors out of a total of 707 trials for the two experimental groups. Further, there was no apparent connection between the number of errors a child made in discrimination training and the adequacy of his drawings on either pre- or posttests. It appears to be the case that for the pointing group our training made some difference. But this does not tell us, if the discriminations were so good to begin with, why there were so many errors of a specific type on the children's pretest drawings. If our overall hypothesis is correct, a child should draw what he is able to discriminate, and our Ss were obviously not doing this. What appears to be the case is that the child cannot draw what he has not discriminated, but he may or may not draw features which he can discriminate.

Thus while the present results lend some support to the "number of attributes" hypothesis, they also suggest that some revision of that hypothesis

Table 1

Mean Number of Specific Errors on Pre- and Posttest Drawings

\begin{tabular}{lccccc} 
Group & $\begin{array}{c}\text { Pretest } \\
\text { drawings }\end{array}$ & $\begin{array}{c}\text { Posttest } \\
\text { drawings }\end{array}$ & Difference & t & p \\
\hline Control & 7.3 & 8.5 & -1.2 & & \\
Point & 9.2 & 6.7 & +2.5 & 2.879 & $>.01$ \\
Trace & 7.3 & 10.5 & -3.2 & 2.441 & $>.05$ \\
\hline
\end{tabular}


is in order. Some of what we observe as a "lag" between perceiving and performing may be due to inadequate discrimination, but some is apparently due also to the child's inability, or unwillingness, to translate what he has discriminated into his copying behavior.

\section{REFERENCES}

BERKO, J., \& BROWN, R. Psycholinguistic research methods. In P. Mussen (Ed.), Handbook of research methods in child development. New York: John Wiley, 1960.

LOVELL, K. A follow-up study of some aspects of the work of Piaget and
Inhelder on the child's conception of space. Brit. $J$. Educ., 1960, 29, 104-117.

MACCOBY, E. E., \& BEE, H. L. Some speculations concerning the lag between perceiving and performing. Child Develpm, 1965, 36, 367-377.

METCALFE, J. An investigation into certain aspects of speech sound discrimination in children. Unpublished doctoral dissertation, Stanford Univ., 1962.

PIAGET, J., \& INHELDER, B. The child's conception of space. London: Routledge \& Kegan Paul, 1956. 